\title{
Streaming Approach to Quadratic Covariation Estimation Using Financial Ultra-High-Frequency Data
}

\author{
Vladimír Holý \\ University of Economics, Prague \\ Winston Churchill Square 4, 13067 Prague 3, Czechia \\ vladimir.holy@vse.cz \\ Petra Tomanová \\ University of Economics, Prague \\ Winston Churchill Square 4, 13067 Prague 3, Czechia \\ petra.tomanova@vse.cz
}

December 17, 2021

\begin{abstract}
We investigate the computational issues related to the memory size in the estimation of quadratic covariation, taking into account the specifics of financial ultra-high-frequency data. In multivariate price processes, we consider both contamination by the market microstructure noise and the non-synchronicity of the observations. We formulate a multi-scale, flat-top realized kernel, non-flat-top realized kernel, pre-averaging and modulated realized covariance estimators in quadratic form and fix their bandwidth parameter at a constant value. This allows us to operate with limited memory and formulate this estimation as a streaming algorithm. We compare the performance of the estimators with fixed bandwidth parameter in a simulation study. We find that the estimators ensuring positive semidefiniteness require much higher bandwidth than the estimators without this constraint.
\end{abstract}

Keywords: Ultra-High-Frequency Data, Market Microstructure Noise, Quadratic Covariation, Streaming Algorithm.

JEL Codes: C32, C58, C63.

\section{Introduction}

In finance, Engle (2000) coined the term ultra-high-frequency data, meaning irregularly spaced time series recorded at the highest possible frequency corresponding to each transaction or change in bid/ask offer. Financial high-frequency time series include stock prices, foreign exchange rates, and commodity prices. The availability of this high-frequency data allows econometricians to construct more precise models, while facing some new challenges.

A key object in financial econometrics is volatility of the price process. A vast number of papers have demonstrated the benefits of using high-frequency data for volatility estimations in various financial applications. In derivative pricing, incorporating high-frequency information into pricing models of options leads to increased profits from option trading (see e.g., Bandi et al., 2008; Corsi et al., 2013; Sanfelici and Uboldi, 2014). In risk management, using high-frequency estimates of volatility as a basis for determining the value-at-risk and expected shortfall increases their precision (see e.g., Brownlees and Gallo, 2010; Žikeš and Baruník, 2015; Bee et al., 2016). In portfolio optimization, daily portfolio rebalancing based on high-frequency estimates of volatility improves portfolio allocations (see e.g., Liu, 2009; Hautsch et al., 2015; Lunde et al., 2016). Especially in the last case, covariance matrices of large dimensions are often required. Furthermore, in algorithmic trading, analyzing highfrequency data is necessary as decisions are also made at high frequencies (see e.g., Christensen et al., 2012; Loveless et al., 2013; Arce et al., 2019). Computationally efficient algorithms for estimating the variance and covariance are therefore crucial for practical use.

For continuous processes, volatility over a given time interval (e.g., a day) is typically measured by the quadratic variation (see e.g., Andersen et al., 2001; Barndorff-Nielsen and Shephard, 2002). 
In the theoretically ideal setting for the price process, it can be straightforwardly estimated by the realized variance. In practice, however, the so-called efficient price is concealed, as the observed prices are contaminated by market microstructure noise, making the realized variance significantly biased at high frequencies. Market microstructure noise is caused by various frictions in the trading process, such as the discretness of the prices, the bid-ask spread, and information effects (see e.g., Hansen and Lunde, 2006).

One way to deal with market microstructure noise is to sample the price process at lower frequencies and find the optimal trade-off between precision and the bias due to this noise (see Aït-Sahalia et al., 2005; Zhang et al., 2005; Bandi and Russell, 2008). A better way is to retain the entire dataset but use estimators that are robust to the noise. There are three dominant approaches in the nonparametric estimation of the quadratic variation: subsampling (see Zhang et al., 2005; Zhang, 2006; Nolte and Voev, 2012; Aït-Sahalia et al., 2011), autocovariance combining (see Barndorff-Nielsen et al., 2008, 2009) and pre-averaging (see Jacod et al., 2009; Hautsch and Podolskij, 2013; Jacod and Mykland, 2015). These estimators have different motivations but very similar structure in the end. Sun (2006) and Andersen et al. (2011) show that the multi-scale estimator of Zhang (2006) based on subsampling and the flat-top realized kernel estimator of Barndorff-Nielsen et al. (2008) based on autocovariance combining can be expressed as a quadratic form. Furthermore, it can be shown that the pre-averaging estimator of Jacod et al. (2009) also has the structure of a quadratic form. Finally, when assuming a specific model for the price process, a parametric approach to estimating the volatility can also be adopted (see Aït-Sahalia et al., 2005, 2010; Xiu, 2010; Holý and Tomanová, 2019).

The estimation of the quadratic covariation between two price processes is even more challenging, due to the non-synchronicity of the trading. When the observations are simply synchronized using the previous-tick interpolation scheme, the Epps effect occurs and the realized covariance is biased (see e.g., Hayashi and Yoshida, 2005; Zhang, 2011). Zhang (2011), however, shows that subsampling in the two-scales estimator cancels not only the market microstructure noise but the Epps effect as well. Hayashi and Yoshida (2005) propose a consistent estimator for the quadratic covariation using the original unaltered data. This approach is also adopted by Palandri (2006), Nolte and Voev (2008), Christensen et al. (2010) and Bibinger (2011). Martens (2004), Christensen et al. (2010) and Barndorff-Nielsen et al. (2011) synchronize the observations using the refresh times of Harris et al. (1995).

Bandi et al. (2008) highlight that a large portion of the high-frequency literature is devoted to the asymptotic properties of the volatility estimators, while the finite-sample properties are given less attention. Nevertheless, there are several studies comparing the finite-sample performance of volatility estimators according to various criteria. Liu et al. (2015) focus on estimation and forecasting accuracy, Gatheral and Oomen (2010) on statistical efficiency, implementation, and robustness, Bandi et al. (2008) with Sanfelici and Uboldi (2014) on their impact on option pricing, and Brownlees and Gallo (2010) on their impact on risk management. The goal of our paper is to compare the volatility estimators from yet another criterion - computational efficiency with a focus on memory constraints.

From a computational point of view, it is natural to consider financial high-frequency data as a data stream. A streaming algorithm can examine a sequence of inputs in a single pass only. The available memory for the computation is limited, and cannot store all the data. Note that different papers have used different definitions of this concept (see e.g., Kontorovich, 2012 vs. Černý, 2019). Related concepts are that of an online algorithm or a recursive algorithm, which focus on the updating scheme rather than memory constraints. Examples of streaming, online, and recursive algorithms in the field of econometrics include the estimation and diagnostics of the linear regression model by Černý (2019), estimation of the ARMA model by Ouakasse and Mélard (2014), estimation of the GARCH model by Aknouche and Guerbyenne (2006), estimation of the EWMA model by Hendrych and Cipra (2019), estimation of the spot volatility by Dahlhaus and Neddermeyer (2014), and detection of changepoints by Bodenham and Adams (2017).

In our paper, we focus on the non-parametric estimation of the quadratic covariation by means of streaming. We consider both the market microstructure noise and the non-synchronicity of the trading. First, we introduce the commonly used estimators that are robust to the market microstructure 
noise: the multi-scale estimator of Zhang (2006), the flat-top realized kernel estimator of BarndorffNielsen et al. (2008), the non-flat-top realized kernel estimator of Barndorff-Nielsen et al. (2011), the pre-averaging estimator of Jacod et al. (2009), and the modulated realized covariance estimator of Christensen et al. (2010). With the aim of providing a unified and simple computational framework, we express the estimators as quadratic forms. All five estimators depend on a bandwidth parameter. These papers show that the optimal value of this parameter depends on the number of observations. Nevertheless, we assume the bandwidth parameter to be constant. This of course leads to sub-optimal performance but allows us to adopt a streaming algorithm with fixed memory, leading to fast computation. In the case of non-synchronous trading, we synchronize the observations by the refresh times method of Harris et al. (1995) with no computational issues.

The main contributions of our paper are the following. First, we express the quadratic covariation estimators as quadratic forms and illustrate the differences in their structure. Second, we propose using a streaming algorithm with fixed bandwidth for estimating the quadratic covariation when the computational performance is an issue. Third, we compare the quadratic covariation estimators and show the impact of the constant bandwidth parameter in a simulation study. We find that the multi-scale estimator is the most precise estimator in the case of small bandwidth, followed by the flat-top realized kernel estimator and the pre-averaging estimator. On the other hand, the non-flattop realized kernel estimator and the modulated realized covariance estimator require much higher bandwidth but ensure positive semidefiniteness.

The rest of this paper is organized as follows. In Section 2, we present the standard framework for the price process and quadratic variation. In Section 3, we describe the class of quadratic estimators and our streaming approach. In Section 4, we evaluate the performance of the estimators with fixed bandwidth using simulations. We conclude the paper in Section 5.

\section{Theoretical Framework}

\subsection{Efficient Price Process}

We use the standard framework for the price process. Denote the $m$-dimensional logarithmic efficient price of a security by $P_{t}$ in continuous time $t \geq 0$. In theory, the efficient price is the true price of a security. In practice, however, the efficient price is unobservable. We assume that the efficient price follows a multivariate continuous Itô semimartingale given by

$$
P_{t}=P_{0}+\int_{0}^{t} \mu_{s} \mathrm{~d} s+\int_{0}^{t} \sigma_{s} \mathrm{~d} W_{s}
$$

where $\mu_{s}$ is a multivariate finite variation càdlàg drift process, $\sigma_{s}$ is a multivariate adapted càdlàg volatility process, and $W_{s}$ is a vector of independent Wiener processes. Note that both $\mu_{s}$ and $\sigma_{s}$ can vary over time and this class is therefore quite general. However, a limitation is that we do not consider jumps, as the process is defined to have continuous paths.

\subsection{Quadratic Covariation}

Our aim is to measure the volatility of the efficient price process over a given time interval. Without loss of generality, we may restrict ourselves to the time interval $[0,1]$. The quadratic covariation of the process $P_{t}$ on $[0,1]$ is then given by

$$
Q V=\operatorname{plim}_{\Delta_{n} \rightarrow 0} \sum_{i=1}^{n}\left(P_{T_{i}}-P_{T_{i-1}}\right)\left(P_{T_{i}}-P_{T_{i-1}}\right)^{\prime},
$$

where plim denotes the limit in probability and $\Delta_{n}=\max \left\{T_{1}-T_{0}, T_{2}-T_{1}, \ldots, T_{n}-T_{n-1}\right\}$ is the maximal lag between observations of synchronized partitions $0=T_{0}<T_{1}<\cdots<T_{n}=1$. As $\Delta_{n} \rightarrow 0$, we have that $n \rightarrow \infty$. In our case of a continuous Itô semimartingale, the quadratic variation is identical to the integrated covariance given by

$$
I V=\int_{0}^{1} \sigma_{s} \sigma_{s}^{\prime} \mathrm{d} s
$$


For general semimartingales, however, they differ, due to the jump component.

\subsection{Observed Price Process}

As noted above, the efficient price is latent. This is for two reasons. First, we cannot observe the process in continuous time, but only at discrete points. Second, the efficient price is concealed due to various microstructural mechanisms, including the discretness of the prices, the bid-ask spread, and information effects. We capture this deviation from the efficient price by an additive noise term, referred to in the literature as the market microstructure noise.

Assume that we observe an $m$-dimensional price process contaminated by the market microstructure noise at non-synchronous discrete times. Denote the $k$-th univariate observed price process by $X_{i}^{k}$ at discrete times $0 \leq T_{0}^{k}<T_{1}^{k}<\cdots<T_{n^{k}}^{k} \leq 1$. Note that the observations can be irregularly spaced. The $k$-th component of the latent price process $P_{t}^{k}$ and the observed price process $X_{i}^{k}$ are then related by

$$
X_{i}^{k}=P_{T_{i}^{k}}^{k}+E_{i}^{k}, \quad E_{i}^{k} \sim\left(0, \omega^{k}\right) \text { for } i=0, \ldots, n^{k},
$$

where $E_{i}^{k}$ is the market microstructure noise; it has expected value zero and standard deviation $\omega^{k}$. At this point, we do not impose additional assumptions on the noise $E_{i}^{k}$, as different estimators require different assumptions. We refer to the papers for the individual estimators for more details. In an empirical study of financial markets, Hansen and Lunde (2006) show that the market microstructure noise is dependent both on its past values and the efficient price process.

\subsection{Data Synchronization}

Recall that we allow different univariate price processes to be observed at different times. In the multivariate setting, this naturally creates difficulties. An elegant approach is to synchronize the observation times, as proposed by Harris et al. (1995). We define the refresh times $0 \leq T_{0}<T_{1}<$ $\cdots<T_{n} \leq 1$ in the following way. Let the initial refresh time be

$$
T_{0}=\max \left\{T_{0}^{1}, \ldots, T_{0}^{m}\right\} .
$$

Next, let the subsequent refresh times be

$$
T_{i}=\min \left\{t: t \geq T_{j^{1}}^{1}>T_{i-1}, t \geq T_{j^{2}}^{2}>T_{i-1}, \ldots, t \geq T_{j^{m}}^{m}>T_{i-1}\right\} \quad \text { for } i=1, \ldots, n .
$$

Martens (2004) uses this scheme for the realized covariance. Barndorff-Nielsen et al. (2011) shows that this synchronization leads to consistent estimates of the quadratic covariation by the realized kernel estimator. Christensen et al. (2010) present similar results for the modulated realized covariance estimator.

After the synchronization, we can write the $m$-dimensional observed price process as $X_{i}=$ $\left(X_{i}^{1}, X_{i}^{2}, \ldots, X_{i}^{m}\right)^{\prime}$ and the market microstructure noise as $E_{i}=\left(E_{i}^{1}, E_{i}^{2}, \ldots, E_{i}^{m}\right)^{\prime}$ with synchronized times $T_{i}, i=0, \ldots, n$. We define the observed returns as $Y_{i}=X_{i}-X_{i-1}, i=1, \ldots, n$. Finally, let $Y=\left(Y_{1}, \ldots, Y_{n}\right)=\left(Y_{i}^{k}\right)_{k=1, i=1}^{m, n}$ denote the matrix of the observed returns with rows corresponding to the assets and columns corresponding to the time. Of course, not all prices are observed exactly at a moment that corresponds to a refresh time. Often, only a single asset has a new price observed. For the other assets, the last observed price is used. This is similar to the previous-tick approach, but the price interpolation is performed only at refresh times instead of all observation times.

\section{Streaming Estimation of Quadratic Variation}

\subsection{Class of Quadratic Estimators}

We estimate the quadratic covariation $Q V$ in the presence of market microstructure noise by various non-parametric methods within a unified framework based on a quadratic form. Sun (2006) and Andersen et al. (2011) consider the class of quadratic estimators in the univariate case of quadratic 
variation. In the multivariate case, the class of quadratic estimators of the quadratic covariation based on the returns $Y_{i}$ are those having the form

$$
Q E=\sum_{i=1}^{n} \sum_{j=1}^{n} w_{i, j} Y_{i} Y_{j}^{\prime}=Y W Y^{\prime},
$$

where $W=\left(w_{i, j}\right)_{i=1, j=1}^{n, n}$ are weights associated with a given estimator. The formula can also be rewritten in terms of the actual prices $X_{i}$ as

$$
Q E=\sum_{i=0}^{n} \sum_{j=0}^{n} v_{i, j} X_{i} X_{j}^{\prime}=X V X^{\prime},
$$

where the weights $V=\left(v_{i, j}\right)_{i=0, j=0}^{n, n}$ are given by $V=F^{\prime} W F$. The elements of $F=\left(f_{i, j}\right)_{i=1, j=0}^{n, n}$ are given by

$$
f_{i, j}= \begin{cases}1 & \text { for } j=i+1 \\ -1 & \text { for } j=i \\ 0 & \text { otherwise }\end{cases}
$$

\subsection{Streaming Approach}

In general, the computation of a quadratic form cannot be formulated as a streaming algorithm, as each observation is required to be stored in the memory. However, we can impose restrictions on the weight matrix $W$ in order to make the computation streaming. Assume the elements of the weight matrix $W$ satisfy

$$
w_{i, j}= \begin{cases}u_{|i-j|} & \text { for }|i-j|<h, \\ 0 & \text { otherwise }\end{cases}
$$

where $u=\left(u_{0}, \ldots, u_{h-1}\right)^{\prime}$ is the updating vector determining a given streaming estimator. The matrix $W$ is therefore a symmetric $(2 h-1)$-diagonal matrix in which the elements in the main diagonal and each lower and upper diagonal are the same and determined by the updating vector $u$. At time $T_{i}$, the quadratic estimator can then be recursively computed by

$$
Q E_{i}=Q E_{i-1}+u_{0} Y_{i} Y_{i}^{\prime}+\sum_{j=1}^{h-1} u_{j} Y_{i} Y_{i-j}^{\prime}+\sum_{j=1}^{h-1} u_{j} Y_{i-j} Y_{i}^{\prime} .
$$

Besides the previous matrix $Q E_{i-1}$ and the vector of the current observations $Y_{i}$, we need to store the vectors of the previous observations $Y_{i-1}, \ldots, Y_{i-h+1}$ in memory. In total, that makes $m(m+h)$ real numbers.

In the following sections, we show that many estimators of the quadratic covariation can be expressed in a quadratic form (7) satisfying the restriction (10) if the bandwidth of the estimators is fixed at $h$ and possible edge effects are neglected. On the one hand, a fixed bandwidth leads to sub-optimal performance of the estimators. On the other hand, it allows estimating the quadratic variation in the presence of market microstructure noise by means of a streaming algorithm. We define the edge effects as the deviations of the weights $w_{i j}$ in the left upper corner $i, j=1, \ldots, h-1$ and the right lower corner $i, j=n-h+2, \ldots, n$ from the values suggested by the updating vector $u$. The multi-scale, pre-averaging and modulated realized covariation estimators possess edge effects (see Figures 4, 7 and 8 respectively) while both the flat-top and non-flat-top realized kernel estimators do not (see Figures 5 and 6 respectively). Jacod et al. (2009) argue that the the edge effects are asymptotically unbiased in the case of the pre-averaging estimator and allow a simpler central limit theorem than in the case of the flat-top realized kernel estimator. In our streaming application, however, it is more suitable to omit the edge effects and modify all considered estimators in the fashion of Barndorff-Nielsen et al. (2008). 
Weight Matrix for Returns

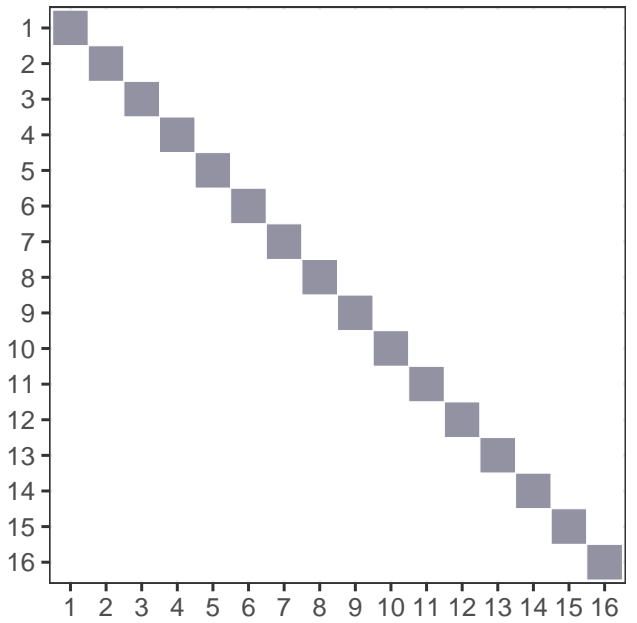

Realized Covariance

Weight Matrix for Prices

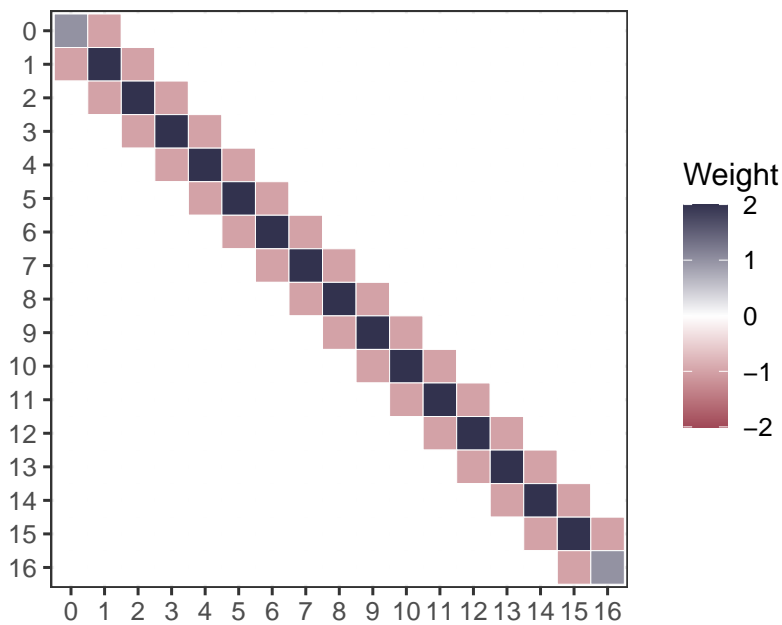

Figure 1: Quadratic form of the realized variance with $n=16$.

\subsection{Realized Covariation}

A natural estimator of the quadratic covariation is the realized covariance, defined by

$$
R V_{n}=\sum_{i=1}^{n} Y_{i} Y_{i}^{\prime}
$$

In the absence of noise, it is a consistent estimator of the quadratic covariation. In the presence of noise, however, it is biased and inconsistent. Note that in the asymptotics as $n \rightarrow \infty$, the time interval remains $[0,1]$ but the frequency of observations increases. The realized covariance can be easily expressed as a quadratic estimator using the weight matrix $W^{R V, n}=I$. An example of this weight matrix is shown in Figure 1 . The updating vector is simply $u^{R V, n}=(1,0, \ldots, 0)^{\prime}$.

\subsection{Multi-Scale Estimator}

The first unbiased and consistent non-parametric estimator of the quadratic variation proposed in the literature is the two-scale estimator of Zhang et al. (2005). It combines the average of realized variances computed at a lower frequency with realized variance at the highest possible frequency. The first term serves as a biased estimate of quadratic variation while the second term estimates the variance of the noise and therefore functions as the bias correction. Zhang (2006) further generalizes the two-scale estimator to a multi-scale estimator based on the average realized variances computed at multiple frequencies. Aït-Sahalia et al. (2011) shows that the multi-scale estimator is robust to serial dependency in the market microstructure noise. In the subsampling spirit, Nolte and Voev (2012) propose combining average realized variances using least squares. Extensions to the estimation of quadratic covariation have been proposed by Palandri (2006), Nolte and Voev (2008), Zhang (2011) and Bibinger (2011).

Before presenting the multi-scale estimator, we need to define some preliminary quantities. First, we introduce the sparse realized covariance, which is simply the realized covariance at a lower frequency. Let $l$ denote the index of the initial observation and $s$ denote the sampling interval. For example $l=1$ and $s=4$ would correspond to observations at times $\left\{T_{1}, T_{5}, T_{9}, T_{13}, \ldots\right\}$. The number of observed prices used in the estimation is then $\lfloor(n-l) / s\rfloor+1$, where $\lfloor\cdot\rfloor$ denotes rounding down. The sparse realized covariance is defined by

$$
S R V_{n, l, s}=\sum_{k=1}^{\lfloor(n-l) / s\rfloor}\left(X_{k s+l}-X_{(k-1) s+l}\right)^{2}=\sum_{k=1}^{\lfloor(n-l) / s\rfloor}\left(\sum_{j=1}^{s} Y_{(k-1) s+l+j}\right)^{2}
$$




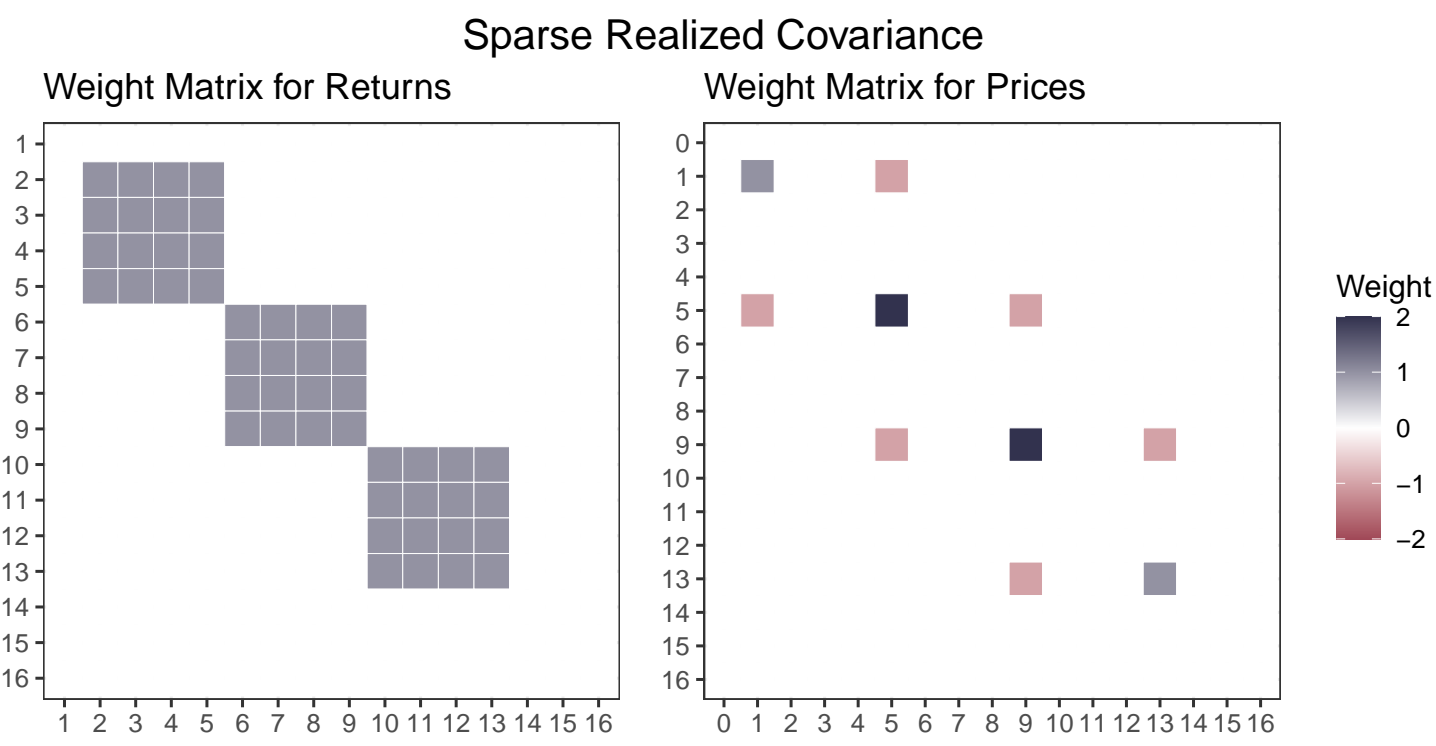

Figure 2: Quadratic form of the sparse realized variance with $n=16, l=1$ and $s=4$.

It can be expressed as a quadratic estimator using the weight matrix $W^{S R V, n, l, s}$ given by

$$
w_{i, j}^{S R V, n, l, s}= \begin{cases}1 & \text { for } i, j \in[(k-1) s+l+1, k s+l], k=1, \ldots,\lfloor(n-l) / s\rfloor, \\ 0 & \text { otherwise }\end{cases}
$$

Figure 2 illustrates the quadratic form. However, the sparse realized covariance cannot be expressed using an updating vector as it does not satisfy the requirements given by (10).

Next, we introduce the average realized covariance. As the sparse realized covariance uses only a fraction of the available observations, it is natural to use all observations by averaging the sparse realized covariances over subgrids given by different $l$. For a given sampling interval $s$, the average realized covariance is defined by

$$
A R V_{n, s}=\frac{1}{s} \sum_{l=0}^{s-1} S R V_{n, l, s} .
$$

Although this approach reduces the impact of the market microstructure noise, the average realized variance is still a biased estimator of the quadratic variation. It can be expressed as a quadratic estimator using the weight matrix

$$
W^{A R V, n, s}=\frac{1}{s} \sum_{l=0}^{s-1} W^{S R V, n, l, s} .
$$

This weight matrix is shown in Figure 3. The updating vector $u^{A R V, n, s}$, omitting edge effects, is given by

$$
u_{i}^{A R V, n, s}=\frac{s-i}{s} \quad \text { for } i=0, \ldots, s-1 .
$$

Finally, we present the multi-scale estimator of Zhang (2006). It is a weighted average of the average realized variances based on the sampling intervals ranging from 1 up to the bandwidth $h$. It is given by

$$
M S E_{n, h}=\sum_{s=1}^{h} A(s, h) A R V_{n, s},
$$

where $A(s, h)$ is a weight function. Zhang (2006) suggests using

$$
A(s, h)=\frac{12 s^{2}}{h^{3}-h}-\frac{6 s}{h^{2}-h} .
$$




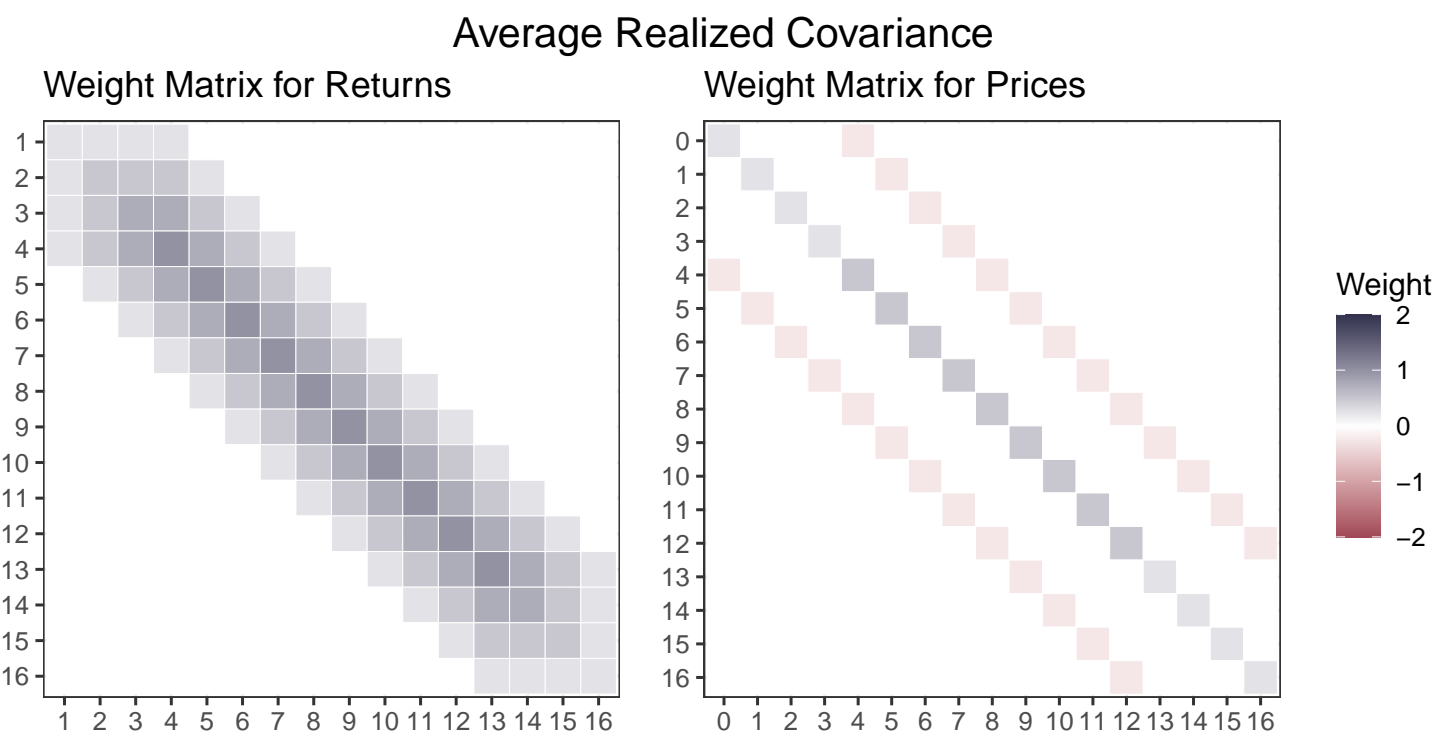

Figure 3: Quadratic form of the average realized variance with $n=16$ and $s=4$.

The weight matrix is given by

$$
W^{M S E, n, h}=\sum_{s=1}^{h} A(s, h) W^{A R V, n, s} .
$$

It is illustrated in Figure 4. The updating vector $u^{M S E, n, h}$ omitting edge effects is given by

$$
u_{i}^{M S E, n, h}=\sum_{s=i+1}^{h} \frac{s-i}{s} A(s, h) \quad \text { for } i=0, \ldots, h-1 .
$$

\subsection{Realized Kernel Estimator}

Another approach for the robust estimation of the quadratic variation is combining realized autocovariances using kernel functions. Barndorff-Nielsen et al. (2008) propose using flat-top kernels with unit weights at lags 0 and 1 . Barndorff-Nielsen et al. (2009) use non-flat-top kernels with unit weight only at lag 0 . Flat-top realized kernels provide a faster convergence rate but do not guarantee the non-negativity of the estimates. In contrast, non-flat-top realized kernels have sub-optimal convergence rates but ensure non-negativity. Barndorff-Nielsen et al. (2011) propose non-flat-top kernels for the estimation of the quadratic covariation.

The flat-top realized kernel estimator of Barndorff-Nielsen et al. (2008) is defined by

$$
R K E_{n, h}=R V_{n}+\sum_{j=1}^{h-1} K\left(\frac{j-1}{h-1}\right)\left(R A_{n, j}+R A_{n,-j}\right),
$$

where $K(\cdot)$ is a kernel function and $R A_{n, l}$ is the realized autocovariance defined by

$$
R A_{n, l}=\sum_{i=l+1}^{n} Y_{i} Y_{i-l}^{\prime} \quad \text { for } l \geq 0
$$

and $R A_{n,-l}^{\prime}$ for $l<0$. Barndorff-Nielsen et al. (2008) consider many kernel functions and find that the modified Tukey-Hanning kernel of order 2 is near efficient. It is defined by

$$
K(x)=\sin ^{2}\left(\frac{\pi}{2}(1-x)^{2}\right) .
$$




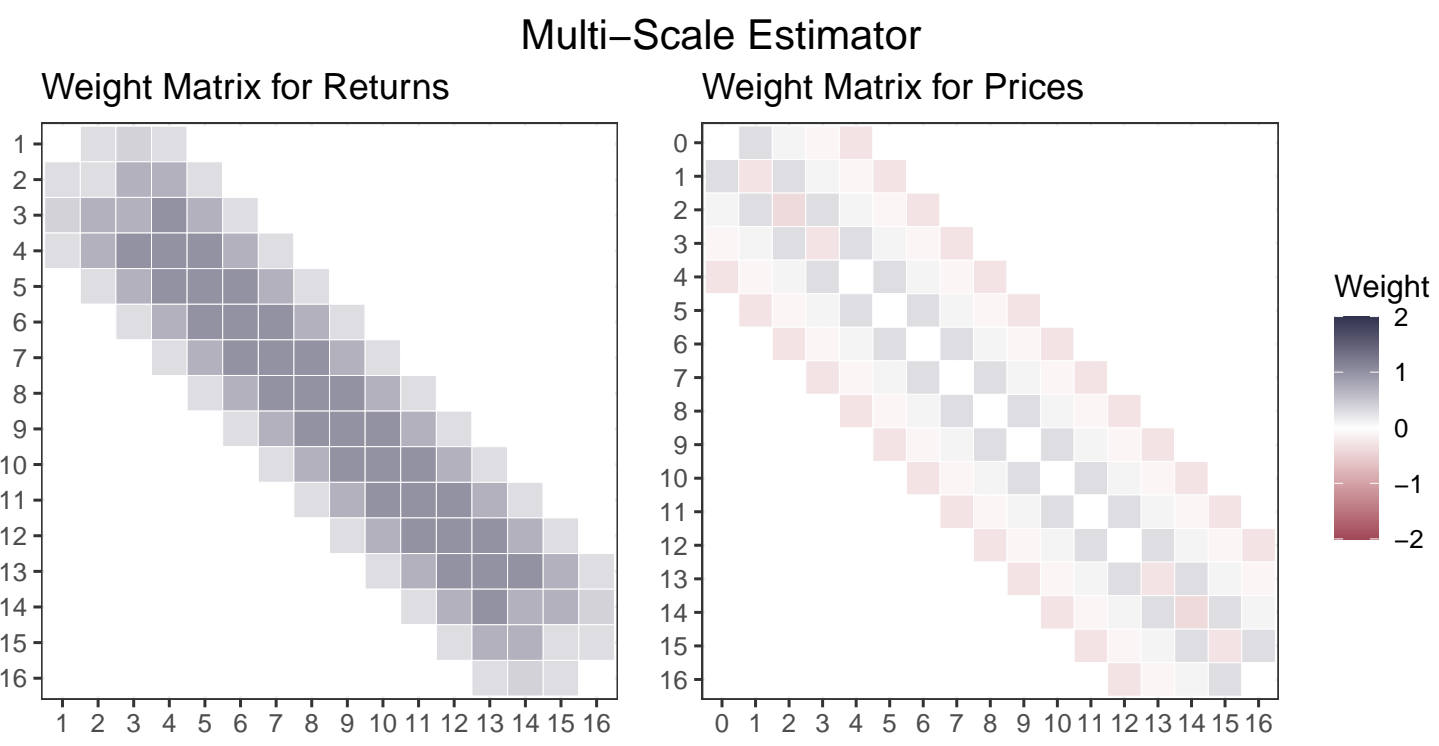

Figure 4: Quadratic form of the multi-scale estimator with $n=16$ and $h=4$.

The flat-top realized kernel estimator can be expressed as a quadratic form with weight matrix $W^{R K E, n, h}$ given by

$$
w_{i, j}^{R K E, n, h}= \begin{cases}1 & \text { for } i=j, \\ K\left(\frac{|i-j|-1}{h-1}\right) & \text { for } 1 \leq|i-j|<h, \\ 0 & \text { otherwise. }\end{cases}
$$

It is illustrated in Figure 5. The updating vector $u^{R K E, n, h}$ is given by

$$
u_{i}^{R K E, n, h}= \begin{cases}1 & \text { for } i=0, \\ K\left(\frac{i-1}{h-1}\right) & \text { for } i=1, \ldots, h-1 .\end{cases}
$$

To ensure a positive semidefinite covariance matrix, the non-flat-top realized kernel of BarndorffNielsen et al. (2011) can be used. It is defined by

$$
P D-R K E_{n, h}=\sum_{j=-h+1}^{h-1} K\left(\frac{|j|}{h}\right) R A_{n, j} .
$$

Both Barndorff-Nielsen et al. (2009) in the univariate case and Barndorff-Nielsen et al. (2011) in the multivariate case suggest using the Parzen kernel given by

$$
K(x)= \begin{cases}1-6 x^{2}+6 x^{3} & \text { for } 0 \leq x<\frac{1}{2} \\ 2(1-x)^{3} & \text { for } \frac{1}{2} \leq x \leq 1\end{cases}
$$

The non-flat-top realized kernel estimator can be expressed as a quadratic form with weight matrix $W^{P D-R K E, n, h}$ given by

$$
w_{i, j}^{P D-R K E, n, h}= \begin{cases}K\left(\frac{|i-j|}{h}\right) & \text { for }|i-j|<h, \\ 0 & \text { otherwise. }\end{cases}
$$

It is illustrated in Figure 6. The updating vector $u^{P D-R K E, n, h}$ is simply given by

$$
u_{i}^{P D-R K E, n, h}=K\left(\frac{i}{h}\right) \quad \text { for } i=0, \ldots, h-1 .
$$


Flat-Top Realized Kernel Estimator

Weight Matrix for Returns

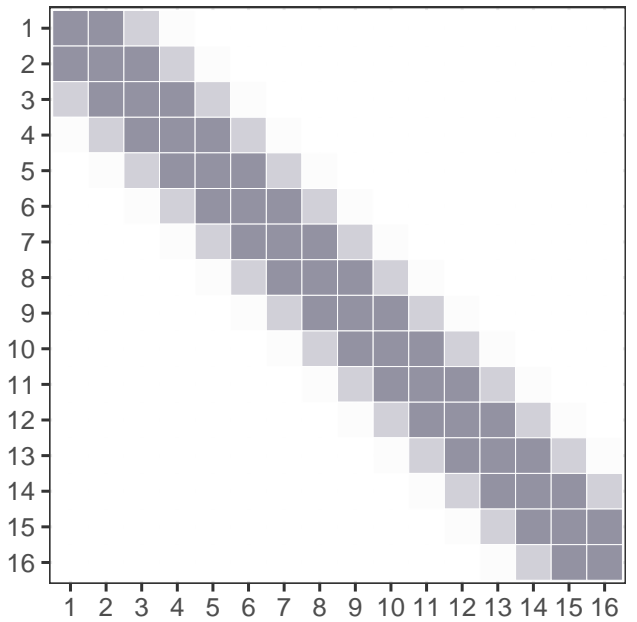

Weight Matrix for Prices

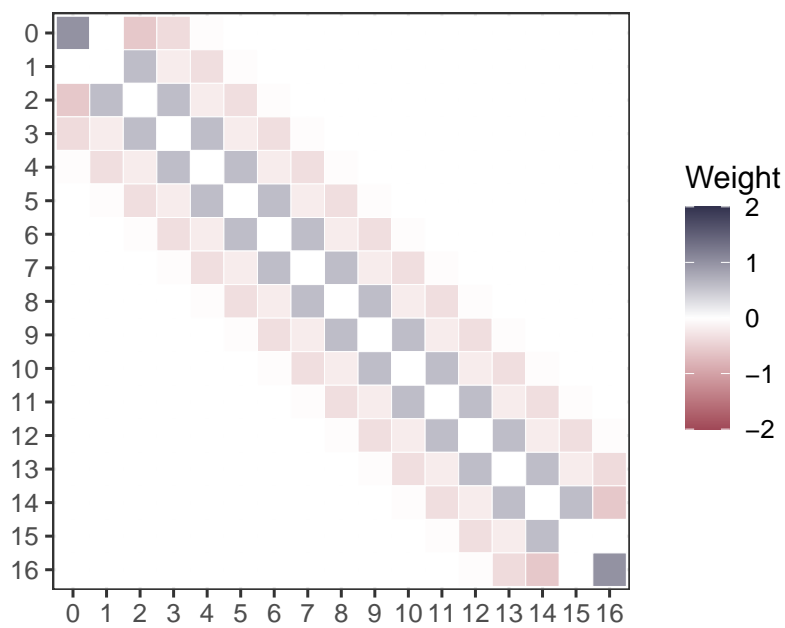

Figure 5: Quadratic form of the flat-top realized kernel estimator with $n=16$ and $h=4$.

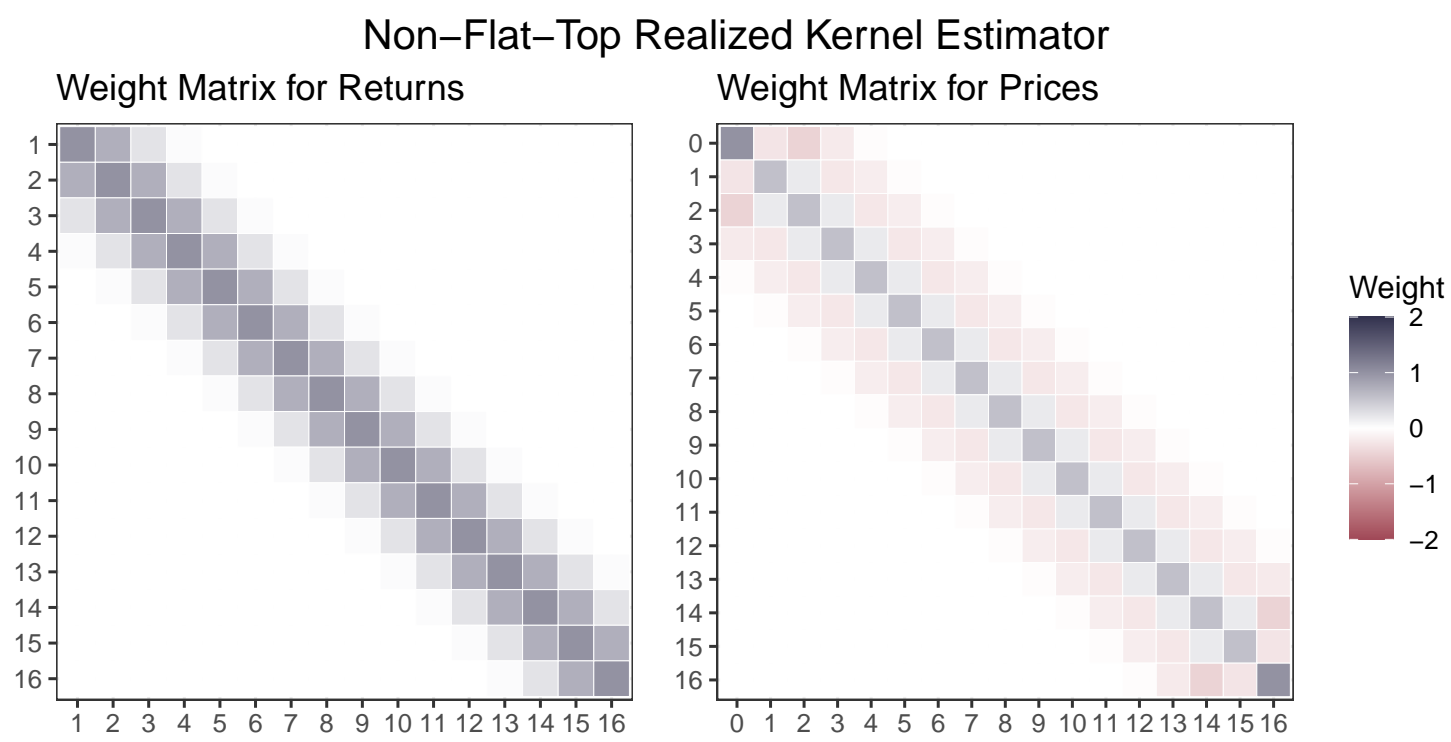

Figure 6: Quadratic form of the non-flat-top realized kernel estimator with $n=16$ and $h=4$. 


\subsection{Pre-Averaging Estimator}

The third class of estimators we present are the pre-averaging estimators pioneered by Jacod et al. (2009). The idea is to locally average the returns and then sum their squares. Hautsch and Podolskij (2013) extend the theory of pre-averaging estimators to accommodate a serial dependence in the market microstructure noise and jumps in the price process. Jacod and Mykland (2015) propose an adaptive method for the choice of the bandwidth parameter. Christensen et al. (2010) extend the pre-averaging estimator to the multivariate setting and use the name modulated realized covariance instead. by

The pre-averaging estimator of Jacod et al. (2009) is based on the averaged returns, and is given

$$
\bar{Y}_{i}=\sum_{j=0}^{h-1} G\left(\frac{j+1}{h+1}\right) Y_{i+j}
$$

where $G(\cdot)$ is a suitable weight function. Jacod et al. (2009) suggest using

$$
G(x)=\min \{x, 1-x\} .
$$

The pre-averaging estimator is then defined by

$$
P A E_{n, h}=\frac{1}{\psi_{2}} \sum_{i=1}^{n-h+1} \bar{Y}_{i} \bar{Y}_{i}^{\prime}-\frac{\psi_{1}}{2 \psi_{2}} R V_{n}
$$

where

$$
\begin{aligned}
& \psi_{1}=\sum_{j=0}^{h}\left(G\left(\frac{j+1}{h+1}\right)-G\left(\frac{j}{h+1}\right)\right)^{2}, \\
& \psi_{2}=\sum_{j=0}^{h-1} G\left(\frac{j+1}{h+1}\right)^{2} .
\end{aligned}
$$

The realized variance term serves as a correction for the bias. Note that similarly to Jacod and Mykland (2015), we use a simpler expression for the estimator than that introduced in Jacod et al. (2009), and omit terms related to the asymptotics of the bandwidth parameter. We also do not include the adjustment for the sample size, as the final number of observations is unknown in the typical streaming setting. The pre-averaging estimator can be expressed as a quadratic estimator using the weight matrix $W^{P A E, n, h}$ given by

$$
w_{i, j}^{P A E, n, h}= \begin{cases}\frac{1}{\psi^{2}} \sum_{k=\max \{0, i+h-n-1\}}^{\min \{h-1, i-1\}} G^{2}\left(\frac{k+1}{h+1}\right)-\frac{\psi_{1}}{2 \psi_{2}} & \text { for } i=j, \\ \frac{1}{\psi^{2}} \sum_{k=\max \{0, i+h \mid,-n-1, j-1\}}^{\min \{-1-\mid i-j+h-n-1\}} G\left(\frac{k+1}{h+1}\right) G\left(\frac{k+1+|i-j|}{h+1}\right) & \text { for } 1 \leq|i-j|<h, \\ 0 & \text { otherwise. }\end{cases}
$$

The weight matrix is illustrated in Figure 7. The updating vector $u^{P A E, n, h}$, omitting the edge effects, is given by

$$
u_{i}^{P A E, n, h}= \begin{cases}\frac{1}{\psi^{2}} \sum_{j=0}^{h-1} G^{2}\left(\frac{j+1}{h+1}\right)-\frac{\psi_{1}}{2 \psi_{2}} & \text { for } i=0, \\ \frac{1}{\psi^{2}} \sum_{j=0}^{h-1-i} G\left(\frac{j+1}{h+1}\right) G\left(\frac{j+1+i}{h+1}\right) & \text { for } i=1, \ldots, h-1 .\end{cases}
$$

When the bias correction term in (33) is omitted, the resulting estimator is guaranteed to be positive semidefinite. Similary to the non-flat-top realized kernel estimator, however, it has a suboptimal convergence rate. Christensen et al. (2010) define the modulated realized covariance estimator by

$$
P D-P A E_{n, h}=\frac{1}{\psi_{2}} \sum_{i=1}^{n-h+1} \bar{Y}_{i} \bar{Y}_{i}^{\prime}
$$




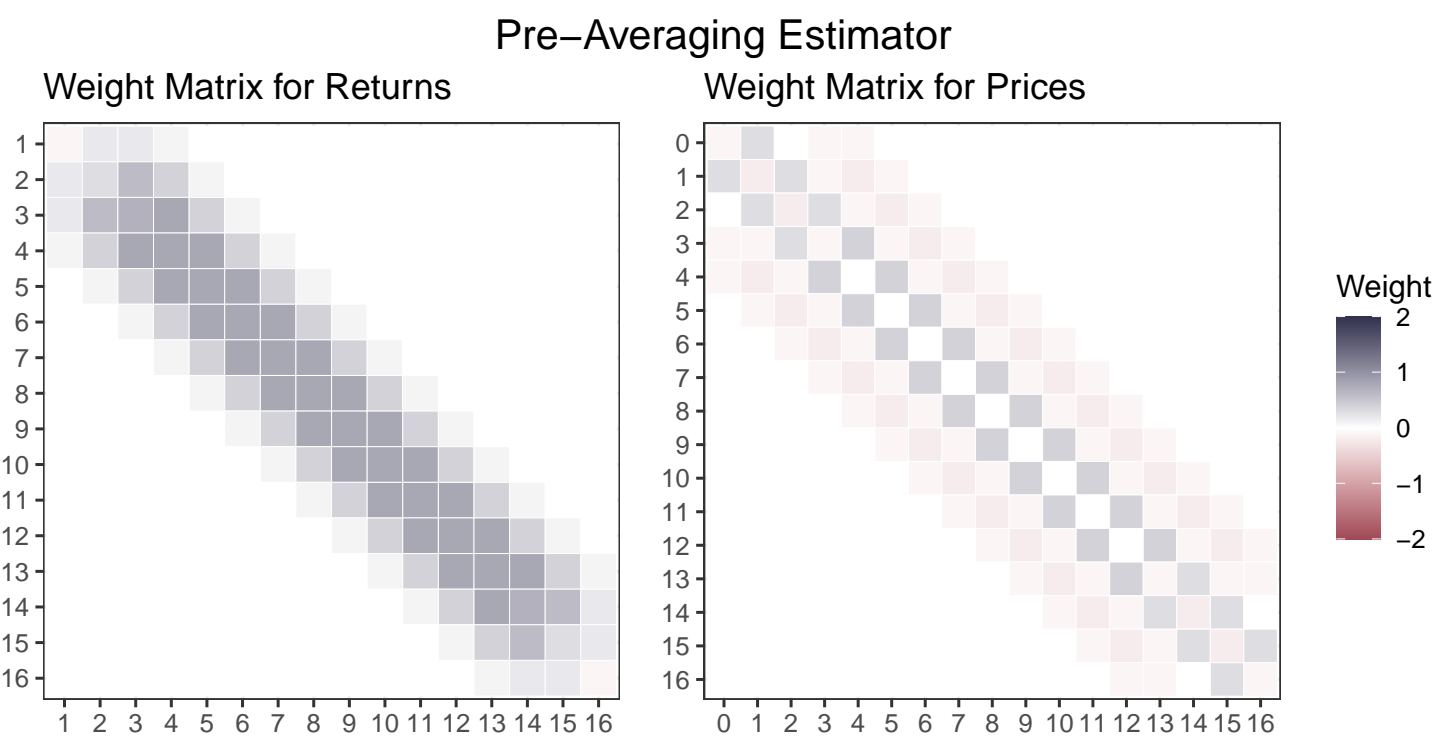

Figure 7: Quadratic form of the pre-averaging estimator with $n=16$ and $h=4$.

The weight matrix $W^{P D-P A E, n, h}$ is given by

$$
w_{i, j}^{P D-P A E, n, h}=\frac{1}{\psi^{2}} \sum_{k=\max \{0, i+h-n-1, j+h-n-1\}}^{\min \{h-1-|i-j|, i-1, j-1\}} G\left(\frac{k+1}{h+1}\right) G\left(\frac{k+1+|i-j|}{h+1}\right) \quad \text { for } 1 \leq|i-j|<h .
$$

This weight matrix is shown in Figure 8. The updating vector $u^{P D-P A E, n, h}$, omitting the edge effects, is given by

$$
u_{i}^{P D-P A E, n, h}=\frac{1}{\psi^{2}} \sum_{j=0}^{h-1-i} G\left(\frac{j+1}{h+1}\right) G\left(\frac{j+1+i}{h+1}\right) \quad \text { for } i=0, \ldots, h-1 .
$$

\section{Simulation Study}

\subsection{Setup for the Simulations}

To compare the finite-sample performance of the estimators, we conducted a simulation study. We consider the same model for the observed price process as Barndorff-Nielsen et al. (2011). The individual efficient price $P^{k}, k=1, \ldots, m$, in continuous time follows

$$
\mathrm{d} P^{k}=\mu \mathrm{d} t+\mathrm{d} V^{k}+\mathrm{d} F^{k},
$$

where $V^{k}$ and the $F^{k}$ are given by

$$
\begin{aligned}
\mathrm{d} V^{k} & =\rho S^{k} \mathrm{~d} B^{k}, \\
\mathrm{~d} F^{k} & =\sqrt{1-\rho^{2}} S^{k} \mathrm{~d} B^{0},
\end{aligned}
$$

and $B_{0}, B_{1}, \ldots, B_{m}$ are independent Wiener processes. The volatility process $S^{k}$ is given by

$$
\begin{aligned}
S^{k} & =\exp \left(\alpha+\beta U^{k}\right), \\
\mathrm{d} U^{k} & =\theta U^{k} \mathrm{~d} t+\mathrm{d} B^{k} .
\end{aligned}
$$

We restrict ourselves to two-dimensional processes, i.e., $m=2$. We generate the observation times $T_{i}^{k}$ by two independent Poisson point processes with scale parameters $\lambda=\left(\lambda_{1}, \lambda_{2}\right)$. We standardize 


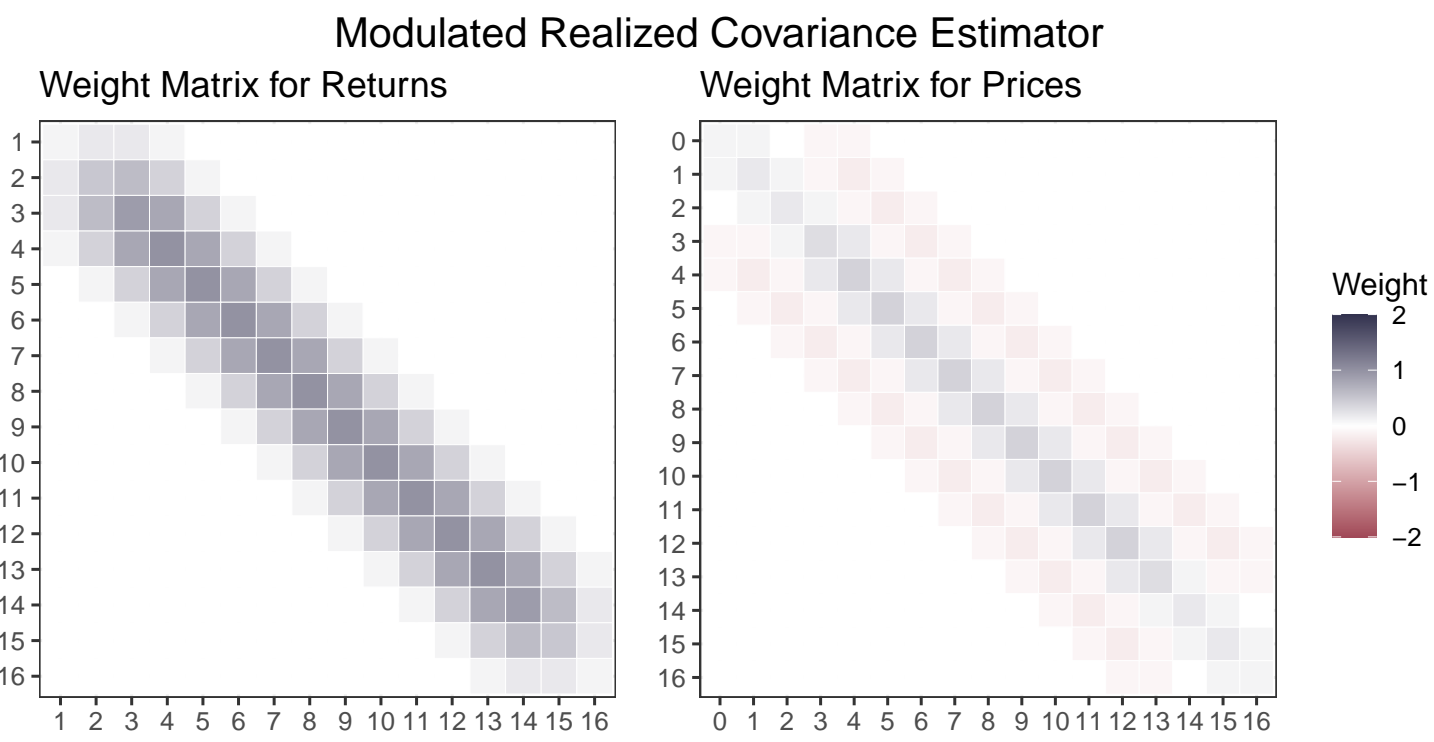

Figure 8: Quadratic form of the modulated realized covariation estimator with $n=16$ and $h=4$.

the time so that one unit corresponds to one second in a trading day that is 6.5 hours long. The time window for which we compute the quadratic covariation is therefore 23400 seconds long. In the case of two independent Poisson processes, the refresh time sampling results in an average number of observations of

$$
n=23400 \frac{\lambda_{1}+\lambda_{2}}{\lambda_{1}^{2}+\lambda_{1} \lambda_{2}+\lambda_{2}^{2}} .
$$

We contaminate the efficient prices by market microstructure noise. The observed prices $X^{k}$ are then

$$
X_{i}^{k}=P_{T_{i}^{k}}^{k}+E_{i}^{k}, \quad E_{i}^{k} \sim \mathrm{N}\left(0, \omega^{k}\right), \quad \omega^{k}=\xi \sqrt[4]{\frac{1}{n+1} \sum_{i=0}^{n}\left(S_{T_{i}^{k}}^{k}\right)^{4}} .
$$

The simulations were perfomed 1000000 times, i.e., we simulated 1000000 days. The volatility process (42) was simulated using the exact simulation algorithm for the Ornstein-Uhlenbeck process. The first observation for each day was generated using the stationary distribution $Q_{0}^{k} \sim$ $\mathrm{N}\left(0,(-2 \theta)^{-1}\right)$. The efficient price process (40) with its components (41) was simulated using the Euler method. The initial observation was set to $P_{0}^{k}=0$.

As in Barndorff-Nielsen et al. (2011), we set the parameter values for the efficient prices to $\mu=0.03, \rho=-0.3, \alpha=-0.3125, \beta=0.125$, and $\theta=-0.025$. These values reflect the empirical properties of financial markets and result in the expected value of quadratic covariation given by

$$
\mathrm{E}[Q V]=\left(\begin{array}{cc}
1.00 & 0.67 \\
0.67 & 1.00
\end{array}\right)
$$

Furthermore, we consider three values for the noise: $\xi^{2}=0$ (referred to as None), $\xi^{2}=0.001$ (referred to as Small) and $\xi^{2}=0.01$ (referred to as Large). We consider two frequencies: $\delta=(1,0.5)$ (referred to as Moderate) and $\delta=(0.1,0.05)$ (referred to as High). We choose much higher frequencies than Barndorff-Nielsen et al. (2011), so as to reflect current trading behavior. The scenarios with moderate frequency have on average 23400 and 46800 observations, respectively, while the high frequency scenarios have 234000 and 468000 observations, respectively. After the refresh time sampling, we have on average 20057 observations when using the moderate frequency and 200571 observations when using the high frequency. 

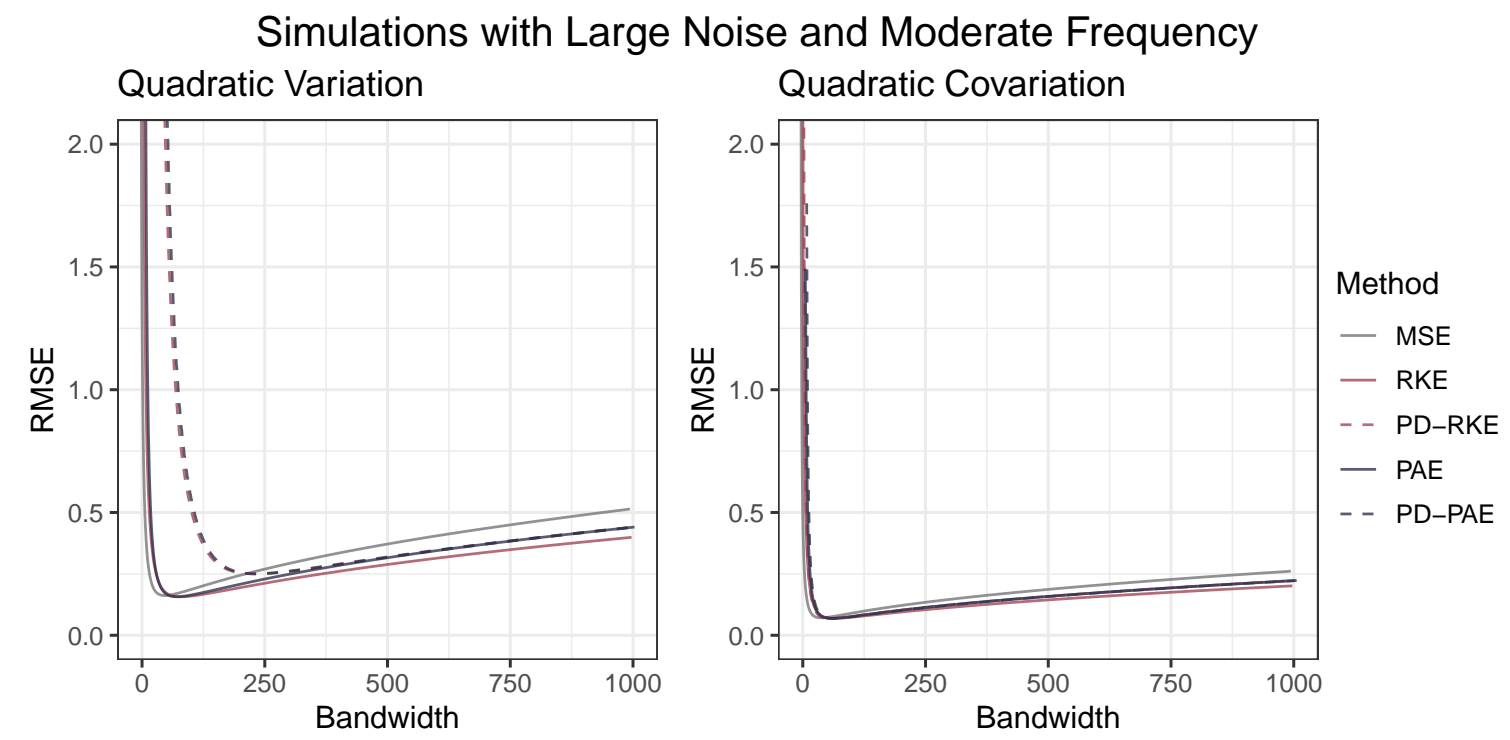

Figure 9: Root-mean-square error of quadratic covariation estimates for various bandwidth parameters when $\xi^{2}=0.01$ and $\delta=(1,0.5)$.

\subsection{Results}

The results of the simulation study are presented in Table 1, Figure 9, and Figure 10. In Table 1 , we choose the best performing bandwidth parameter according to the root-mean-square error. In Figures 9 and 10, we investigate the behavior of the root-mean-square error for values of the bandwidth parameter ranging from 2 to 1000 (regardless of the number of observations).

The realized variance is the best estimator in the univariate case when there is no market microstructure noise. When noise is present, however, the realized variance is significantly biased with increasing frequency. In the multivariate case, the realized covariance is negatively biased under the refresh time sampling. These are both standard results well covered in the literature.

The multi-scale, flat-top realized kernel and pre-averaging estimators perform comparably. They have lower root-mean-square error of the univariate estimates than the non-flat-top realized kernel and modulated realized covariance estimators in the presence of market microstructure noise. However, they do not ensure positive semidefiniteness, which is a major drawback. Table 1 with Figures 9 and 10 show that the multi-scale estimator requires the lowest bandwidth for the optimal performance and is the best choice when the bandwidth is lower than optimal. As this is the natural situation in the streaming setting, we recommend adopting the multi-scale estimator in practice when memory is limited. When the bandwidth is higher than optimal, however, the flat-top realized kernel estimator is the most precise. The pre-averaging estimator represents a middle way.

The non-flat-top realized kernel and modulated realized covariance estimators ensure positive semidefiniteness. On the other hand, they are less precise and require higher bandwidth in the univariate case, as shown in Table 1 and Figures 9 and 10. In the case of the quadratic covariation between two series, however, they are quite comparable to the estimators not ensuring positive semidefiniteness. Interestingly, the non-flat-top realized kernel estimator has almost the same performance as the modulated realized covariance estimator, even though their structures differ, as illustrated in Figures 6 and 8. As requiring a positive semidefinite covariance matrix is quite a reasonable requirement, either of these two methods should be used when the bandwidth parameter is not limited.

\section{Conclusion}

We have dealt with the estimation of the quadratic covariation, taking into account the specifics of financial ultra-high-frequency data. Namely, we have considered both market microstructure noise 


\begin{tabular}{|c|c|c|c|c|c|c|c|c|}
\hline \multicolumn{3}{|c|}{ Simulation Scenario } & \multicolumn{3}{|c|}{ Quadratic Variation } & \multicolumn{3}{|c|}{ Quadratic Covariation } \\
\hline Noise & Freq. & Method & Band. & Bias & RMSE & Band. & Bias & RMSE \\
\hline None & Mod. & RV & 1 & -0.00 & 0.02 & 1 & -0.19 & 0.27 \\
\hline None & Mod. & MSE & 2 & -0.00 & 0.04 & 2 & -0.00 & 0.02 \\
\hline None & Mod. & RKE & 2 & -0.00 & 0.04 & 2 & -0.00 & 0.02 \\
\hline None & Mod. & PD-RKE & 2 & -0.00 & 0.03 & 11 & -0.01 & 0.03 \\
\hline None & Mod. & PAE & 18 & -0.02 & 0.07 & 18 & -0.01 & 0.03 \\
\hline None & Mod. & PD-PAE & 2 & -0.00 & 0.03 & 10 & -0.01 & 0.03 \\
\hline None & High & $\mathrm{RV}$ & 1 & -0.00 & 0.01 & 1 & -0.19 & 0.27 \\
\hline None & High & MSE & 2 & -0.00 & 0.01 & 2 & -0.00 & 0.01 \\
\hline None & High & RKE & 2 & -0.00 & 0.01 & 2 & -0.00 & 0.01 \\
\hline None & High & PD-RKE & 2 & -0.00 & 0.01 & 18 & -0.00 & 0.01 \\
\hline None & High & PAE & 28 & -0.01 & 0.03 & 28 & -0.00 & 0.01 \\
\hline None & High & PD-PAE & 2 & -0.00 & 0.01 & 16 & -0.00 & 0.01 \\
\hline Small & Mod. & $\mathrm{RV}$ & 1 & 40.25 & 75.13 & 1 & -0.19 & 0.44 \\
\hline Small & Mod. & MSE & 17 & 0.00 & 0.09 & 14 & -0.00 & 0.04 \\
\hline Small & Mod. & RKE & 26 & 0.00 & 0.09 & 22 & -0.00 & 0.04 \\
\hline Small & Mod. & PD-RKE & 85 & 0.04 & 0.14 & 18 & -0.00 & 0.04 \\
\hline Small & Mod. & PAE & 22 & -0.01 & 0.08 & 20 & -0.01 & 0.04 \\
\hline Small & Mod. & PD-PAE & 84 & 0.04 & 0.14 & 17 & -0.00 & 0.04 \\
\hline Small & High & $\mathrm{RV}$ & 1 & 395.54 & 738.56 & 1 & -0.19 & 1.11 \\
\hline Small & High & MSE & 75 & 0.01 & 0.05 & 45 & -0.00 & 0.02 \\
\hline Small & High & RKE & 108 & 0.01 & 0.05 & 69 & -0.00 & 0.02 \\
\hline Small & High & PD-RKE & 348 & 0.02 & 0.09 & 57 & -0.00 & 0.02 \\
\hline Small & High & PAE & 114 & 0.01 & 0.06 & 58 & -0.00 & 0.02 \\
\hline Small & High & PD-PAE & 346 & 0.02 & 0.09 & 57 & -0.00 & 0.02 \\
\hline Large & Mod. & $\mathrm{RV}$ & 1 & 402.48 & 751.65 & 1 & -0.20 & 3.49 \\
\hline Large & Mod. & MSE & 51 & 0.02 & 0.16 & 44 & -0.00 & 0.07 \\
\hline Large & Mod. & RKE & 82 & 0.02 & 0.16 & 68 & -0.00 & 0.07 \\
\hline Large & Mod. & PD-RKE & 228 & 0.07 & 0.25 & 56 & -0.00 & 0.07 \\
\hline Large & Mod. & PAE & 67 & 0.02 & 0.16 & 56 & -0.00 & 0.07 \\
\hline Large & Mod. & PD-PAE & 228 & 0.07 & 0.25 & 55 & -0.00 & 0.07 \\
\hline Large & High & $\mathrm{RV}$ & 1 & 3955.40 & 7385.83 & 1 & -0.20 & 10.75 \\
\hline Large & High & MSE & 232 & 0.03 & 0.11 & 137 & -0.00 & 0.04 \\
\hline Large & High & RKE & 331 & 0.03 & 0.10 & 218 & -0.00 & 0.04 \\
\hline Large & High & PD-RKE & 958 & 0.05 & 0.17 & 181 & -0.00 & 0.04 \\
\hline Large & High & PAE & 348 & 0.03 & 0.11 & 181 & -0.00 & 0.04 \\
\hline Large & High & PD-PAE & 956 & 0.05 & 0.17 & 179 & -0.00 & 0.04 \\
\hline
\end{tabular}

Table 1: Bias and root-mean-square error of quadratic covariation estimates for the best performing bandwidth parameter in various combinations of noise and frequency. 

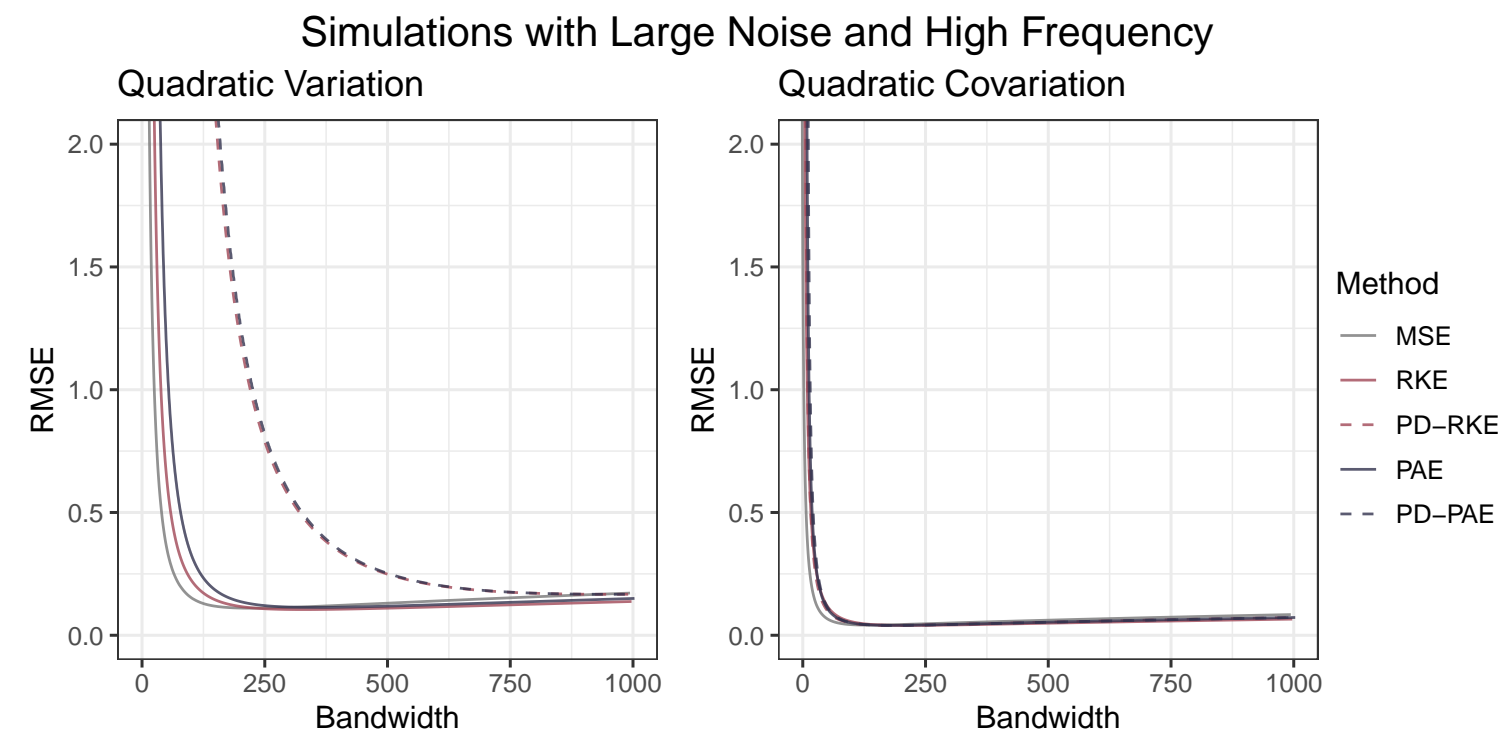

Figure 10: Root-mean-square error of quadratic covariation estimates for various bandwidth parameters when $\xi^{2}=0.01$ and $\delta=(0.1,0.05)$.

and non-synchronous observations. To our knowledge, the high-frequency literature lacks a comprehensive overview of quadratic covariation estimators in a unified framework. We have remedied this and presented the multi-scale, flat-top realized kernel, non-flat-top realized kernel, pre-averaging and modulated realized covariance estimators as quadratic forms. We have also illustrated the differences in their structures. This is the first contribution of the paper.

We have approached the problem of estimating quadratic covariation from a computational point of view, focusing on limited memory. We have used the convenient quadratic structure and shown that the estimates can be computed by a streaming algorithm when the bandwith is fixed and edge effects are neglected. The streaming representation of the estimators is crucial, especially when the covariance matrix is large. This is the second contribution of the paper.

Using simulations, we have compared the finite-sample performance of the estimators with fixed bandwidth. We find that for small bandwidths, the multi-scale estimator is the most precise. The flat-top realized kernel and pre-averaging estimators, however, perform very similarly. In contrast, the non-flat-top realized kernel and modulated realized covariance estimators, which ensure positive semidefiniteness, require much higher bandwidth than the estimators without such a constraint. This is the third contribution of the paper.

Our results can find a use in the implementation of the quadratic covariation estimators in practice. Financial applications include derivative pricing, risk management, portfolio allocation, and highfrequency trading.

\section{Acknowledgements}

We would like to thank the organizers and participants of the 23rd International Conference on Computational Statistics (Iasi, August 28-39, 2018) for fruitful discussions. Computational resources were supplied by the project "e-Infrastruktura CZ" (e-INFRA CZ ID:90140) supported by the Ministry of Education, Youth and Sports of the Czech Republic.

\section{Funding}

This research was supported by the Internal Grant Agency of the University of Economics, Prague under project F4/21/2018 and the Czech Science Foundation under project 19-02773S. 


\section{References}

Aït-Sahalia, Y., Mykland, P. A., Zhang, L. 2005. How Often to Sample a Continuous-Time Process in the Presence of Market Microstructure Noise. The Review of Financial Studies. Volume 18. Issue 2. Pages 351-416. ISSN 0893-9454. https://doi.org/10.1093/rfs/hhi016.

Aït-Sahalia, Y., Fan, J., Xiu, D. 2010. High-Frequency Covariance Estimates with Noisy and Asynchronous Financial Data. Journal of the American Statistical Association. Volume 105. Issue 492. Pages 1504-1517. ISSN 0162-1459. https://doi.org/10.1198/jasa.2010.tm10163.

AïtT-Sahalia, Y., Mykland, P. A., Zhang, L. 2011. Ultra High Frequency Volatility Estimation with Dependent Microstructure Noise. Journal of Econometrics. Volume 160. Issue 1. Pages 160-175. ISSN 0304-4076. https://doi.org/10.1016/j.jeconom.2010.03.028.

Aknouche, A., Guerbyenne, H. 2006. Recursive Estimation of GARCH Models. Communications in Statistics - Simulation and Computation. Volume 35. Issue 4. Pages 925-938. ISSN 0361-0918. https://doi.org/10.1080/03610910600880328.

Andersen, T. G., Bollerslev, T., Diebold, F. X., Labys, P. 2001. The Distribution of Realized Exchange Rate Volatility. Journal of the American Statistical Association. Volume 96. Issue 453. Pages 42-55. ISSN 0162-1459. https://doi.org/10.1198/016214501750332965.

Andersen, T. G., Bollerslev, T., Meddahi, N. 2011. Realized Volatility Forecasting and Market Microstructure Noise. Journal of Econometrics. Volume 160. Issue 1. Pages 220-234. ISSN 0304-4076. https://doi.org/10.1016/j.jeconom.2010.03.032.

Arce, P., Antognini, J., Kristuanpoller, W., Salinas, L. 2019. Fast and Adaptive Cointegration Based Model for Forecasting High Frequency Financial Time Series. Computational Economics. Volume 54. Issue 1. Pages 99-112. ISSN 0927-7099. https://doi .org/10.1007/s10614-017-9691-7.

Bandi, F. M., Russell, J. R. 2008. Microstructure Noise, Realized Variance, and Optimal Sampling. Review of Economic Studies. Volume 75. Issue 2. Pages 339-369. ISSN 0034-6527. https: //doi.org/10.1111/j.1467-937X.2008.00474.x.

Bandi, F. M., Russell, J. R. 2011. Market Microstructure Noise, Integrated Variance Estimators, and the Accuracy of Asymptotic Approximations. Journal of Econometrics. Volume 160. Issue 1. Pages 145-159. ISSN 0304-4076. https://doi.org/10.1016/j.jeconom.2010.03.027.

Bandi, F. M., Russell, J. R., YAng, C. 2008. Realized Volatility Forecasting and Option Pricing. Journal of Econometrics. Volume 147. Issue 1. Pages 34-46. ISSN 0304-4076. https://doi.org/ $10.1016 / j \cdot j$ jeconom.2008.09.002.

Barndorff-Nielsen, O. E., Shephard, N. 2002. Econometric Analysis of Realized Volatility and Its Use in Estimating Stochastic Volatility Models. Journal of the Royal Statistical Society: Series B (Methodological). Volume 64. Issue 2. Pages 253-280. ISSN 1369-7412. https://doi.org/10. 1111/1467-9868.00336.

Barndorff-Nielsen, O. E., Hansen, P. R., Lunde, A., Shephard, N. 2008. Designing Realized Kernels to Measure the ex post Variation of Equity Prices in the Presence of Noise. Econometrica. Volume 76. Issue 6. Pages 1481-1536. ISSN 0012-9682. https://doi.org/10.3982/ecta6495.

Barndorff-Nielsen, O. E., Hansen, P. R., Lunde, A., Shephard, N. 2009. Realized Kernels in Practice: Trades and Quotes. Econometrics Journal. Volume 12. Issue 3. Pages 1-32. ISSN 1368-4221. https://doi.org/10.1111/j.1368-423X.2008.00275.x.

Barndorff-Nielsen, O. E., Hansen, P. R., Lunde, A., Shephard, N. 2011. Multivariate Realised Kernels: Consistent Positive Semi-Definite Estimators of the Covariation of Equity Prices with Noise and Non-Synchronous Trading. Journal of Econometrics. Volume 162. Issue 2. Pages 149-169. ISSN 0304-4076. https://doi.org/10.1016/j.jeconom.2010.07.009. 
Bee, M., Dupuis, D. J., Trapin, L. 2016. Realizing the Extremes: Estimation of Tail-Risk Measures from a High-Frequency Perspective. Journal of Empirical Finance. Volume 36. Pages 86-99. ISSN 0927-5398. https://doi.org/10.1016/j.jempfin.2016.01.006.

Bibinger, M. 2011. Efficient Covariance Estimation for Asynchronous Noisy High-Frequency Data. Scandinavian Journal of Statistics. Volume 38. Issue 1. Pages 23-45. ISSN 0303-6898. https: //doi.org/10.1111/j.1467-9469.2010.00712.x.

Bodenham, D. A., Adams, N. M. 2017. Continuous Monitoring for Changepoints in Data Streams Using Adaptive Estimation. Statistics and Computing. Volume 27. Issue 5. Pages 1257-1270. ISSN 0960-3174. https://doi.org/10.1007/s11222-016-9684-8.

Brownlees, C. T., Gallo, G. M. 2010. Comparison of Volatility Measures: A Risk Management Perspective. Journal of Financial Econometrics. Volume 8. Issue 1. Pages 29-56. ISSN 1479-8417. https://doi.org/10.1093/jjfinec/nbp009.

ČECH, F., BARuník, J. 2017. On the Modelling and Forecasting of Multivariate Realized Volatility: Generalized Heterogeneous Autoregressive (GHAR) Model. Journal of Forecasting. Volume 36. Issue 2. Pages 181-206. ISSN 0277-6693. https://doi.org/10.1002/for.2423.

Černý, M. 2019. Narrow Big Data in a Stream: Computational Limitations and Regression. Information Sciences. Volume 486. Pages 379-392. ISSN 0020-0255. https://doi.org/10.1016/j . ins.2019.02.052.

Christensen, H. L., Murphy, J., Godsill, S. J. 2012. Forecasting High-Frequency Futures Returns Using Online Langevin Dynamics. IEEE Journal on Selected Topics in Signal Processing. Volume 6. Issue 4. Pages 366-380. ISSN 1932-4553. https://doi.org/10.1109/jstsp.2012.2191532.

Christensen, K., Kinnebrock, S., Podolskij, M. 2010. Pre-Averaging Estimators of the Ex-Post Covariance Matrix in Noisy Diffusion Models with Non-Synchronous Data. Journal of Econometrics. Volume 159. Issue 1. Pages 116-133. ISSN 0304-4076. https://doi.org/10.1016/j. jeconom.2010.05.001.

Corsi, F., Fusari, N., La Vecchia, D. 2013. Realizing Smiles: Options Pricing with Realized Volatility. Journal of Financial Economics. Volume 107. Issue 2. Pages 284-304. ISSN 0304-405X. https://doi.org/10.1016/j.jfineco.2012.08.015.

Dahlhaus, R., Neddermeyer, J. C. 2014. Online Spot Volatility-Estimation and Decomposition with Nonlinear Market Microstructure Noise Models. Journal of Financial Econometrics. Volume 12. Issue 1. Pages 174-212. ISSN 1479-8409. https://doi.org/10.1093/jjfinec/nbt008.

Harris, F. H. B., McInish, T. H., Shoesmith, G. L., Wood, R. A. 1995. Cointegration, Error Correction, and Price Discovery on Informationally Linked Security Markets. Journal of Financial and Quantitative Analysis. Volume 30. Issue 4. Pages 563-579. ISSN 0022-1090. https://doi. org $/ 10.2307 / 2331277$.

Engle, R. F. 2000. The Econometrics of Ultra-High-Frequency Data. Econometrica. Volume 68. Issue 1. Pages 1-22. ISSN 0012-9682. https://doi.org/10.1111/1468-0262.00091.

Gatheral, J., Oomen, R. C. A. 2010. Zero-Intelligence Realized Variance Estimation. Finance and Stochastics. Volume 14. Issue 2. Pages 249-283. ISSN 0949-2984. https://doi.org/10.1007/ s00780-009-0120-1.

Hansen, P. R., Lunde, A. 2006. Realized Variance and Market Microstructure Noise. Journal of Business \& Economic Statistics. Volume 24. Issue 2. Pages 127-161. ISSN 0735-0015. https: //doi.org/10.1198/073500106000000071. 
Hautsch, N., PodolskiJ, M. 2013. Preaveraging-Based Estimation of Quadratic Variation in the Presence of Noise and Jumps: Theory, Implementation, and Empirical Evidence. Journal of Business \& Economic Statistics. Volume 31. Issue 2. Pages 165-183. ISSN 0735-0015. https: //doi.org/10.1080/07350015.2012.754313.

Hautsch, N., Kyj, L. M., Malec, P. 2015. Do High-Frequency Data Improve High-Dimensional Portfolio Allocations? Journal of Applied Econometrics. Volume 30. Issue 2. Pages 263-290. ISSN 1099-1255. https://doi.org/10.1002/jae.2361.

Hayashi, T., Yoshida, N. 2005. On Covariance Estimation of Non-Synchronously Observed Diffusion Processes. Bernoulli. Volume 11. Issue 2. Pages 359-379. ISSN 1350-7265. https://doi.org/10. $3150 / \mathrm{bj} / 1116340299$.

Hendrych, R., Cipra, T. 2019. Recursive Estimation of the Exponentially Weighted Moving Average Model. Journal of Risk. Volume 21. Issue 6. Pages 43-67. ISSN 1465-1211. https: //doi.org/10.21314/jor.2019.413.

Holý, V., Tomanová, P. 2019. Estimation of Ornstein-Uhlenbeck Process Using Ultra-HighFrequency Data with Application to Intraday Pairs Trading Strategy. Working Paper. https: //arxiv.org/abs/1811.09312.

JaCOD, J., Mykland, P. A. 2015. Microstructure Noise in the Continuous Case: Approximate Efficiency of the Adaptive Pre-Averaging Method. Stochastic Processes and Their Applications. Volume 125. Issue 8. Pages 2910-2936. ISSN 0304-4149. https://doi.org/10.1016/j.spa.2015. 02.005 .

Jacod, J., Li, Y., Mykland, P. A., PodolskiJ, M., Vetter, M. 2009. Microstructure Noise in the Continuous Case: The Pre-Averaging Approach. Stochastic Processes and Their Applications. Volume 119. Issue 7. Pages 2249-2276. ISSN 0304-4149. https://doi.org/10.1016/j.spa. 2008. 11.004 .

Kontorovich, L. 2012. Statistical Estimation with Bounded Memory. Statistics and Computing. Volume 22. Issue 5. Pages 1155-1164. ISSN 0960-3174. https://doi.org/10.1007/ s11222-011-9293-5.

Liu, L. Y., Patton, A. J., Sheppard, K. 2015. Does Anything Beat 5-Minute RV? A Comparison of Realized Measures Across Multiple Asset Classes. Journal of Econometrics. Volume 187. Issue 1. Pages 293-311. ISSN 1872-6895. https://doi.org/10.1016/j.jeconom.2015.02.008.

Liu, Q. 2009. On Portfolio Optimization: How and When Do We Benefit from High-Frequency Data? Journal of Applied Econometrics. Volume 24. Issue 4. Pages 560-582. ISSN 0883-7252. https://doi.org/10.2307/40206292.

Loveless, J., Stoikov, S., Waeber, R. 2013. Online Algorithms in High-Frequency Trading. Communications of the ACM. Volume 56. Issue 10. Pages 50-56. ISSN 0001-0782. https: //doi.org/10.1145/2507771.2507780.

Lunde, A., Shephard, N., Sheppard, K. 2016. Econometric Analysis of Vast Covariance Matrices Using Composite Realized Kernels and Their Application to Portfolio Choice. Journal of Business 86 Economic Statistics. Volume 34. Issue 4. Pages 504-518. ISSN 0735-0015. https://doi.org/ $10.1080 / 07350015.2015 .1064432$.

Martens, M. P. 2004. Estimating Unbiased and Precise Realized Covariances. Working Paper. https://ssrn. com/abstract=556118.

Nolte, I., Voev, V. 2008. Estimating High-Frequency Based (Co-) Variances: A Unified Approach. Working Paper. https://ssrn. com/abstract=1003201. 
Nolte, I., Voev, V. 2012. Least Squares Inference on Integrated Volatility and the Relationship Between Efficient Prices and Noise. Journal of Business 83 Economic Statistics. Volume 30. Issue 1. Pages 94-108. ISSN 0735-0015. https://doi.org/10.1080/10473289.2011.637876.

Ounkasse, A., MÉlard, G. 2014. On-Line Estimation of ARMA Models Using Fisher-Scoring. Systems Science \& Control Engineering. Volume 2. Issue 1. Pages 406-432. ISSN 2164-2583. https://doi.org/10.1080/21642583.2014.912572.

PAlandri, A. 2006. Consistent Realized Covariance for Asynchronous Observations Contaminated by Market Microstructure Noise. Working Paper. https://ssrn. com/abstract=2727826.

Sanfelici, S., Uboldi, A. 2014. Assessing the Quality of Volatility Estimators via Option Pricing. Studies in Nonlinear Dynamics and Econometrics. Volume 18. Issue 2. Pages 103-124. ISSN 1081-1826. https://doi.org/10.1515/snde-2012-0075.

Sun, Y. 2006. Best Quadratic Unbiased Estimators of Integrated Variance in the Presence of Market Microstructure Noise. Working Paper. https://ssrn.com/abstract=1714751.

XIU, D. 2010. Quasi-Maximum Likelihood Estimation of Volatility with High Frequency Data. Journal of Econometrics. Volume 159. Issue 1. Pages 235-250. ISSN 0304-4076. https://doi. org/10.1016/j.jeconom.2010.07.002.

Zhang, L. 2006. Efficient Estimation of Stochastic Volatility Using Noisy Observations: A MultiScale Approach. Bernoulli. Volume 12. Issue 6. Pages 1019-1043. ISSN 1350-7265. https: //doi.org/10.2307/25464852.

ZHANG, L. 2011. Estimating Covariation: Epps Effect, Microstructure Noise. Journal of Econometrics. Volume 160. Issue 1. Pages 33-47. ISSN 0304-4076. https://doi.org/10.1016/j. jeconom. 2010.03 .012$.

Zhang, L., Mykland, P. A., AïT-Sahalia, Y. 2005. A Tale of Two Time Scales: Determining Integrated Volatility with Noisy High-Frequency Data. Journal of the American Statistical Association. Volume 100. Issue 472. Pages 1394-1411. ISSN 0162-1459. https://doi.org/10.2307/27590680.

Žımeš, F., BARuník, J. 2015. Semi-Parametric Conditional Quantile Models for Financial Returns and Realized Volatility. Journal of Financial Econometrics. Volume 14. Issue 1. Pages 185-226. ISSN 1479-8417. https://doi.org/10.1093/jjfinec/nbu029. 